\title{
Development of Photoactive Supramolecular Devices and Machines
}

\author{
S. P. Gromov ${ }^{a, b}$, *, A. K. Chibisov ${ }^{a}$, and M. V. Alfimov ${ }^{a}$ \\ a Photochemistry Center, Federal Research Center "Crystallography and Photonics," \\ Russian Academy of Sciences, Moscow, 119421 Russia \\ ${ }^{b}$ Moscow State University, Moscow, 119991 Russia \\ *e-mail: spgromov@mail.ru
}

Received October 1, 2020; revised October 1, 2020; accepted October 20, 2020

\begin{abstract}
Among the various directions of the science of photoactive organic compounds, the design, creation, and study of photoactive supramolecular devices and machines are attracting special attention, since in this way it is possible to discover new approaches to construct complex hierarchical "smart" materials.
\end{abstract}

Keywords: trans-cis-photoisomerization, [2+2]-photocycloaddition, charge transfer, photoinduced electron transfer, unsaturated compounds, crown compounds, cucurbiturils, molecular devices, molecular machines, molecular meccano

DOI: $10.1134 / \mathrm{S} 1990793121020202$

It has now become obvious that the main direction of the development of the technosphere of civilization is related to the miniaturization of devices and machines. There are two main strategies for creating nano-sized architectures: bottom-up and top-down. In a top-down strategy, nanoparticles and nano-sized systems are produced by grinding larger objects. The bottom-up strategy can be implemented, first of all, with the help of organic synthesis and supramolecular self-assembly.

A new direction has emerged in nanotechnology from the bottom up: the engineering of supramolecular devices and machines for various purposes. Structurally organized and functionally integrated chemical systems are called supramolecular devices. Supramolecular machines usually include devices in which the implementation of a function occurs as a result of the mechanical movement of components relative to each other. Both the former and the latter can be used to create devices for the generation, conversion, and transfer of energy, and devices for movement at the nanoscale levels, as well as in the development of tools for the monitoring and diagnostics of nanoquantities of materials and substances.

Supramolecular devices and machines can be controlled in different ways (for example, using thermal, chemical, or electrochemical effects), but the most convenient agent is light, which is easily adjustable in both wavelength and intensity.

In living nature, photoactive supramolecular systems provide photosynthesis and phototropism in plants, visual perception in humans and animals, and phototaxis in bacteria: absorbing a photon, chlorophyll and retinal molecules are rearranged, as a result of which their reactivity changes. In these systems, light activates the mechanisms of a number of vital processes. The main photoprocesses that occur in this case are fluorescence, intercombinational transition to the triplet state, energy and electron transfer, and the structural changes caused by light:

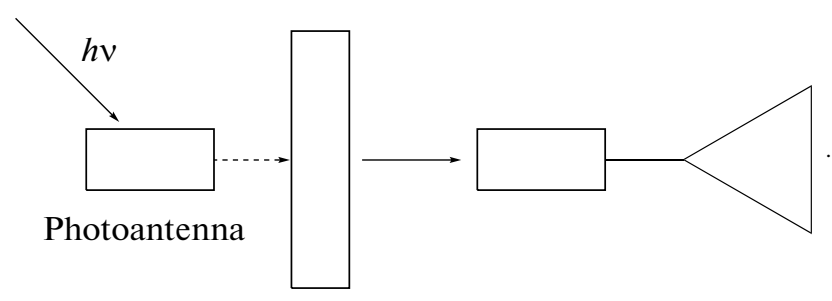

Functional block

Thus, the molecules that make up photoactive supramolecular systems must absorb light, which leads to a structural rearrangement and a change in reactivity. The ability of photoactive supramolecular systems to possess the properties of photoantennas is important.

For a long time attempts were made to create artificial photoactive supramolecular systems of varying degrees of complexity, which, on the one hand, would possess the most valuable properties of natural objects, and on the other, would provide opportunities for new applications. Photochromic derivatives of anthracene and spiro compounds were studied as photoantennas in supramolecular devices and machines; however, azobenzene derivatives containing an $\mathrm{N}=\mathrm{N}$ bond were most often used. However, the fundamental disadvantage of azobenzenes is that they are only capable of entering into trans-cis- and cis-trans-photoisomerization reactions: 

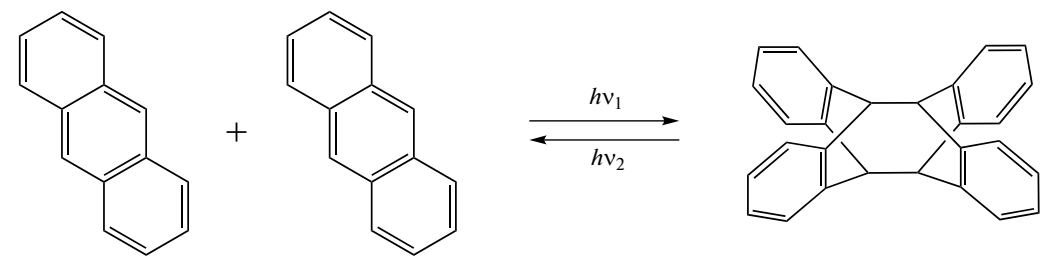<smiles>CN1c2ccccc2C(C)(C)C12C=Cc1cc([N+](=O)[O-])ccc1O2</smiles>
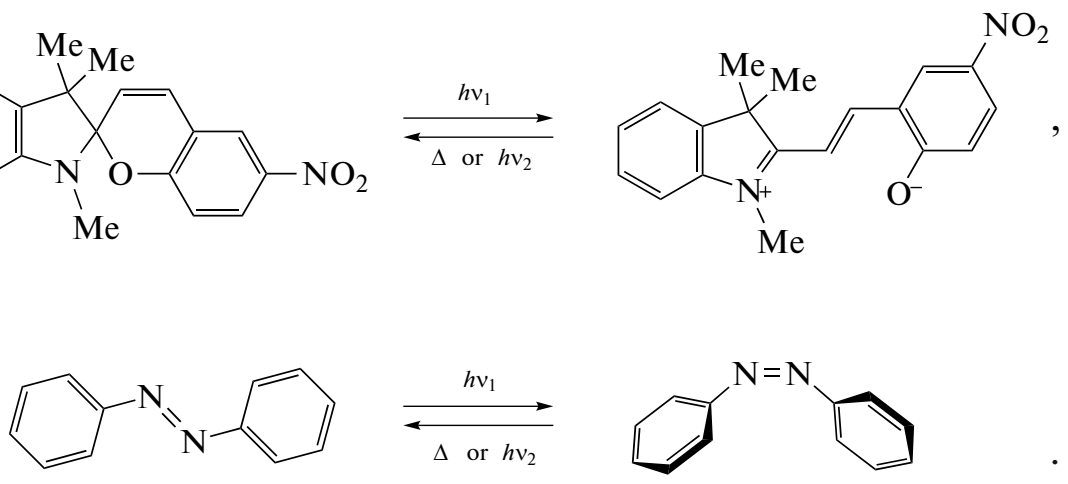

In this respect, compounds containing a carboncarbon double bond (unsaturated compounds) have a number of advantages, the main one of which is the ability to enter not only a photoisomerization reaction but also other reversible photoreactions leading to significant structural changes, for example, in the $[2+2]$-photocycloaddition reaction with the formation of cyclobutane derivatives:

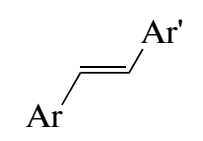

trans-isomer
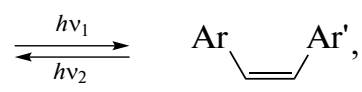

cis-isomer
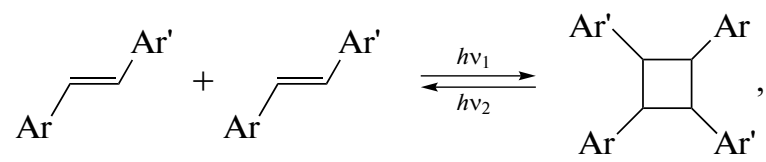

Cyclobutane derivative

Crown compounds are most often used as a functional block (fragments) reacting to a change in the structure of a molecule upon photoswitching. These are macrocyclic compounds containing heteroatoms with lone electron pairs that are capable of forming coordination bonds with a metal ion. One of the fundamental properties of these molecules is their ability in solutions to self-assemble together with ions or other molecules (guests) into systems of a high degree of complexity, which strongly depends on the chemical and spatial structure:

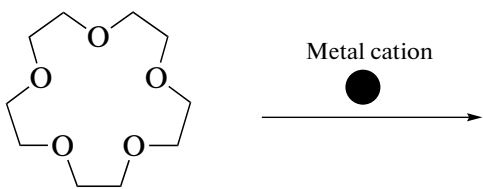

Crown ether<smiles>C1CCCCCCCC1</smiles>

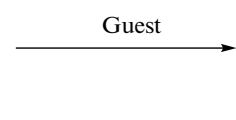

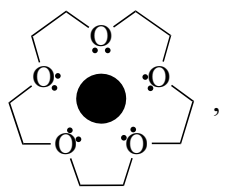

Complex

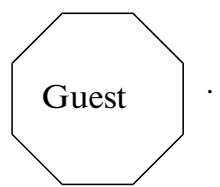

If the fragments of the crown compound and the photoactive compound are linked by covalent bonds in such a way that one or two heteroatoms of the macrocycle are paired with a chromophore, this will lead to a violation of additivity in the manifestation of the specific properties of crown-containing photoactive compounds. In this case, the transformations of the photoactive fragment under the action of light will lead to a change in the efficiency of the metal cations being bound by the crown ether fragment, and self-assembly, in turn, should change the spectral and photochemical characteristics of the photoactive fragment. Conjugated reactions of this type occur in supramolecular structures formed as a result of self-assembly with the participation of molecules of the crown-containing photoactive compound and metal ions, which is used to develop and create photoswitchable supramolecular devices.

Thus, photoswitchable supramolecular devices based on unsaturated compounds and crown compounds can be schematically represented as follows (Fig. 1): they are hybrid molecules containing an unsaturated fragment as a photoantenna and one or 
two crown ether fragments capable of binding to a metal ion [1]. In this case, self-assembly into supramolecular structures will make it possible to change the spectral and photochemical characteristics of the unsaturated fragment of the molecule, and with the help of light, in turn, it will be possible to control the self-assembly of such crown-containing molecules.

The prototypes of photocontrolled supramolecular machines, in which the components can mechanically move relative to each other (Fig. 2), can be shuttle-like pseudorotaxane complexes with the participation of unsaturated compounds (axes) and macrocyclic cucurbiturils or cyclodextrins (rotors). To do this, they should not be connected by strong chemical bonds.

Cucurbiturils, which are used as a rotor in pseudorotaxane complexes, are macrocyclic compounds of the type $\left(\mathrm{C}_{6} \mathrm{H}_{6} \mathrm{~N}_{4} \mathrm{O}_{2}\right)_{n}$, and their structure resembles a pumpkin (in Latin, cucurbita) or a barrel. The size of their internal cavity allows the inclusion of "guests"organic molecules with the formation of complexes. The oxygen atoms of strongly polarized carbonyl groups are located in the regions of the bottom and lid of such a barrel:

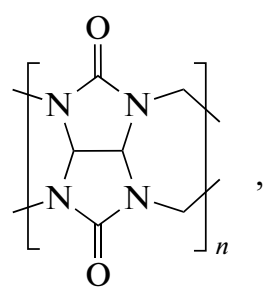

Cucurbiturils

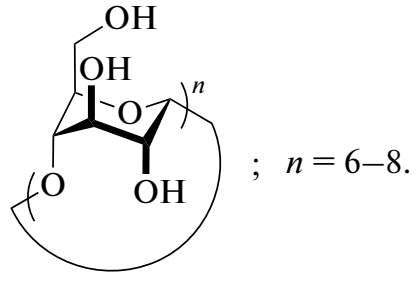

Cyclodextrins
This facilitates the formation of inclusion complexes, especially with positively charged guest molecules, which include most photoactive unsaturated compounds.

The shape of the molecules of cyclodextrins is a torus, which also resembles a hollow truncated cone. This form is stabilized by hydrogen bonds between $\mathrm{OH}$ groups and between $\alpha$-D-1,4-glycosidic linkages. All $\mathrm{OH}$ groups in cyclodextrins are located on the outer surface of the molecule. Therefore, the internal cavity of cyclodextrins is hydrophobic and is capable of forming inclusion complexes with photoactive organic molecules in aqueous solutions.

In living nature, a huge variety of supramolecular architectures are created based on the limited sets of components. Examples of such molecular meccano constructors are nucleotides, which are used in nature for the construction of nucleic acids of various degrees of hierarchy.

The use of the molecular meccano methodology can become a universal technique for constructing photoactive supramolecular devices and machines with the given architecture and different properties in nanotechnology from the bottom up. However, such

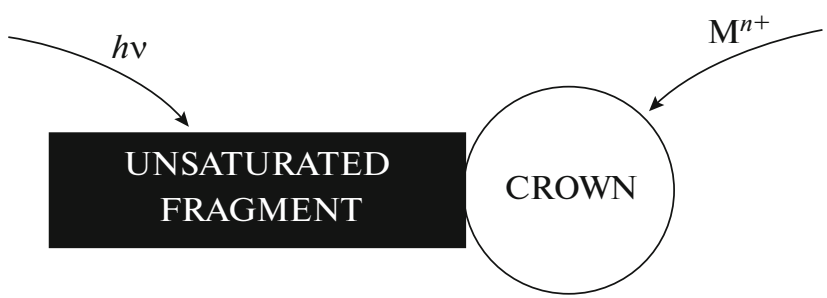

Fig. 1. Schematic representation of photoswitchable supramolecular devices based on crown-containing unsaturated compounds (CUCs).

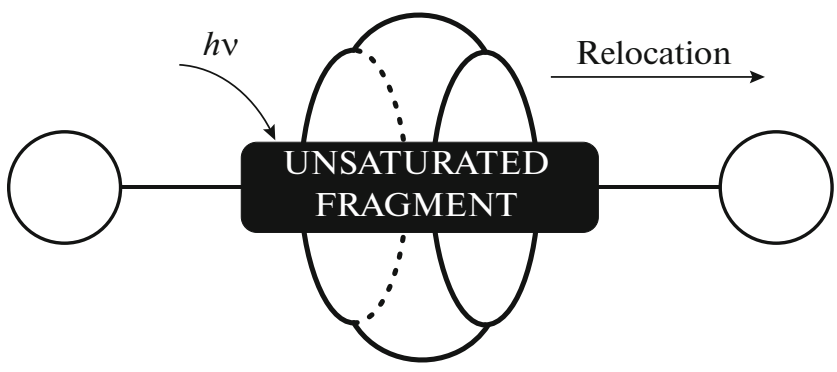

Fig. 2. Schematic representation of photocontrolled supramolecular machines based on unsaturated compounds and cucurbiturils or cyclodextrins.

approaches in nanotechnology have not been developed.

Crown-containing unsaturated compounds (CUCs), which have a $\mathrm{C}=\mathrm{C}$ bond with aryl and hetaryl residues as photoantenna, were synthesized and studied [2]. This made it possible to carry out the photoswitching of molecules using visible light. At the same time, methods were developed for the synthesis of a number of compounds that were not described earlier, but are very promising for various types of photoactive supramolecular devices: derivatives of crown ethers, including hard-to-reach ones, for example, containing $\mathrm{N}, \mathrm{O}$, and $\mathrm{S}$ atoms in the macrocycle in various combinations. It could be assumed that the self-assembly of CUC molecules and metal cations would result in complex supramolecular structures: supramolecular complexes, which also have the ability to photoswitch.

It was found that crown-containing styryl dyes having a terminal anionic group $\mathrm{SO}_{3}^{-}$capable of coordination are the most promising ones for the creation of photoswitchable supramolecular devices. By selfassembly, it was possible to obtain supramolecular complexes from these dyes and metal cations: 

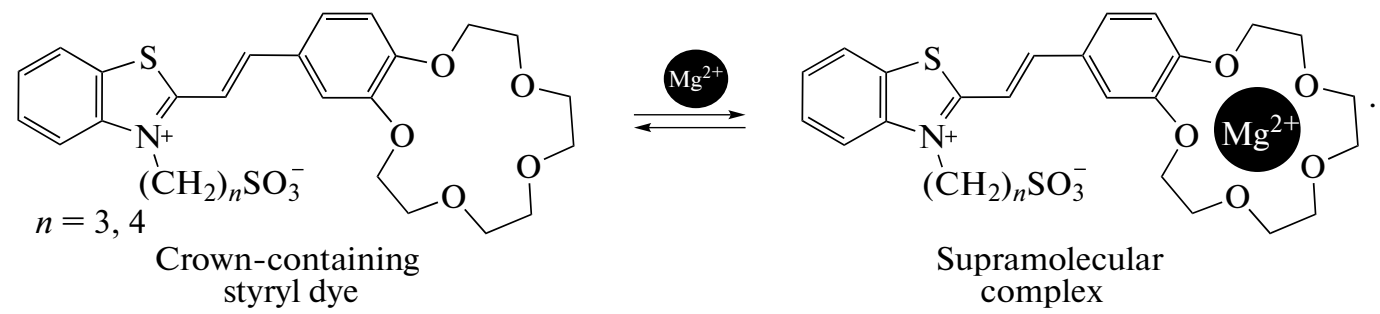

Supramolecular complex

Depending on the wavelength of actinic light, such complexes can "put on" and "take off" their anionic "cap" ( $\mathrm{SO}_{3}^{-}$group); i.e., they are capable of supramolecular photoswitching [3]:

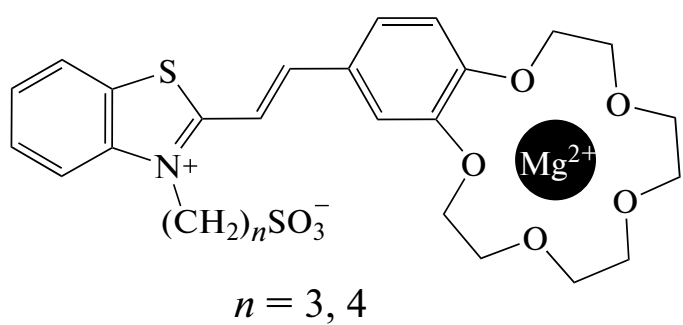

It was also shown that cis-isomers of the abovementioned styryl dyes form much stronger complexes than trans-isomers (the ratio of the stability constant reaches 500). This means that with the help of light it is possible to change the affinity of these CUCs for a metal cation, i.e., manage the process of complex formation. Such supermolecules are commonly called photoswitchable supramolecular devices. Thus, photoswitchable supramolecular devices were obtained for the first time based on the crown-containing styryl dyes. CUC-based supramolecular complexes create possibilities for creating more complex photoswitchable supramolecular devices.

Judging by the data of photochemical studies, in the presence of metal cations, the molecules of crowncontaining styryl dyes are capable of self-assembly into photoactive sandwich structures (head-to-tail dimers), in which the $\mathrm{C}=\mathrm{C}$ bonds are located one above the other. If the solutions of supramolecular dimeric complexes are irradiated with light with a certain wavelength, then the photocycloaddition reaction with the formation of cyclobutane derivatives occurs efficiently [4]:

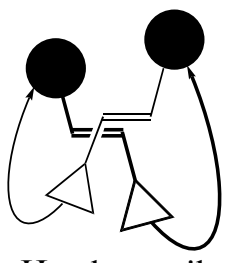

Head-to-tail dimeric complex
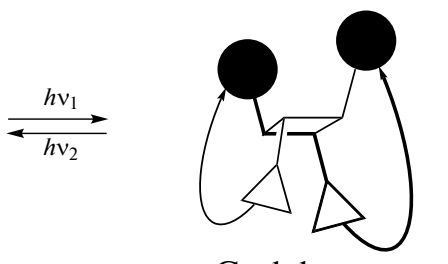

Cyclobutane complex
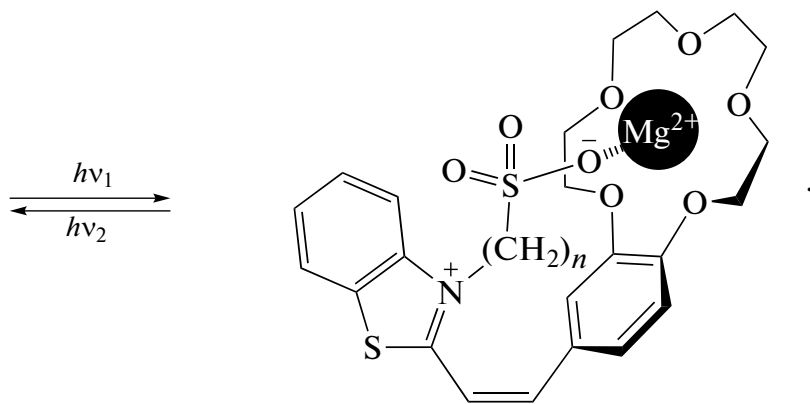

Supramolecular photoswitching
The following notation is used here:

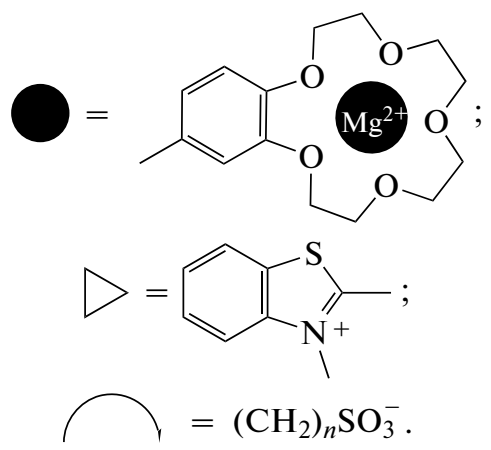

If the cation is removed, then the action of light with a shorter wavelength can convert cyclobutane into the original styryl dye, and then repeat the process of forming the dimeric complex. In addition, it was found that the resulting cyclobutane derivatives bind the metal cation much better than the initial dyes. Thus, in this case, it is possible to control the formation of the complex with the help of light; i.e., dimeric complexes are also photoswitchable supramolecular devices [1].

It should be noted that as a result of supramolecular photocycloaddition, only one of the 11 theoretically possible isomers of cyclobutane is formed. In other words, this reaction proceeds stereospecifically [5]. This makes it possible to purposefully create photoswitchable supramolecular devices of a strictly defined structure.

The data obtained at present allow us to state that metal cations in dimeric complexes of CUCs have no direct effect on the course of the photocycloaddition reaction. They act as molecular glue, bringing molecules closer together to a distance at which the reac- 
tion becomes possible, and at the same time providing a favorable mutual orientation of the $\mathrm{C}=\mathrm{C}$ bonds for the reaction.

In contrast to complexes with an anionic "cap" (anion-covered complexes), the products of photocycloaddition-crown-containing cyclobutanes without metal ions-represent a new type of photoswitchable host molecules with two binding sites for cations. The crown ether fragments in these molecules are arranged in such a way that one can foresee the possibility of their simultaneous participation in the formation of a sandwich-type complex with a large-diameter metal cation, for example, $\mathrm{Ba}^{2+}$ or $\mathrm{Cs}^{+}$, or with a small organic cation.

Thus, photoswitchable supramolecular devices with the specified characteristics can be obtained from two CUC molecules and two metal cations by selfassembly in a solution of supramolecular dimeric complexes with subsequent photoconversion to crown-containing cyclobutanes with different complexing properties. If necessary, cyclobutanes can be disassembled into their initial compounds by irradiation with light with a shorter wavelength [1].

It was of interest to investigate how the presence of an additional $\mathrm{C}=\mathrm{C}$ bond in crown-containing butadienyl dyes affects their complexing and photochemical properties. Among butadienyl dyes (BDs) was discovered an example of the simplest photoswitchable supramolecular device with a switching time of $20 \mathrm{ps.}$ In the $\mathrm{BD} \cdot \mathrm{Ca}^{2+}$ complex, the metal cation has coordination bonds with all the heteroatoms of the crown ether fragment; however, upon photoexcitation of the complex, the calcium-nitrogen bond breaks and reforms after deactivation of the excited state. Thus, in this photoswitchable supramolecular device, the function of the calcium-nitrogen bond formation is controlled:

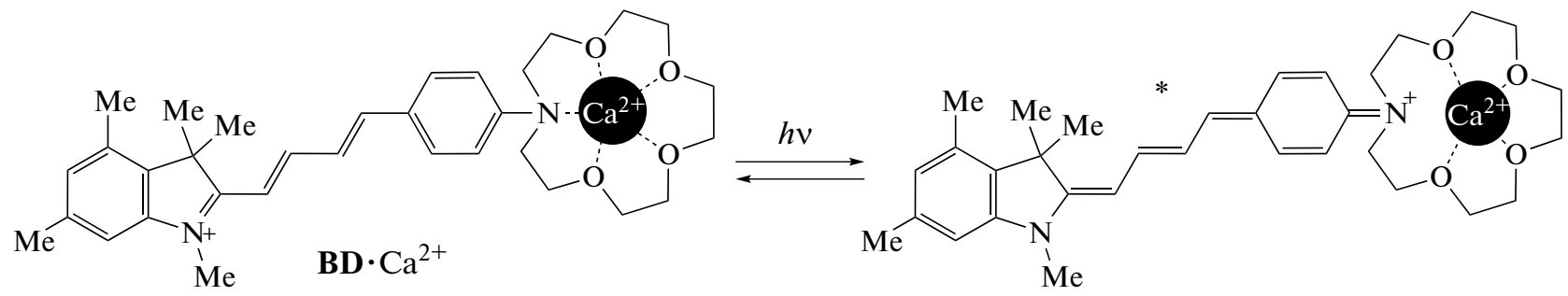

Photoinduced reaction of recoordination

A supramolecular device characterized by photoswitchable intramolecular complexation involving hydrogen bonds in the absence of metal cations was obtained by introducing a long $\mathrm{N}$-ammonium alkyl substituent in the heterocyclic residue of the styryl dye:

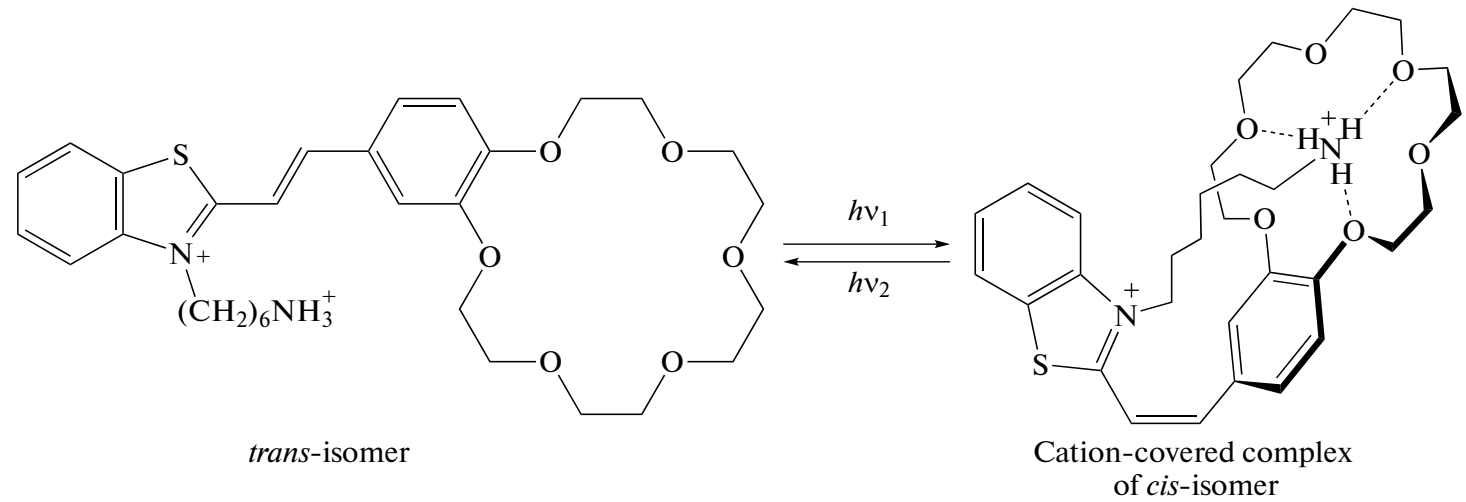

Unlike complexes with a cationic cap (cation-covered complexes), styryl dyes with shorter ammonium alkyl substituents at the nitrogen atom of the heterocyclic residue are capable of forming dimeric complexes in solution by spontaneous supramolecular selfassembly involving hydrogen bonds:

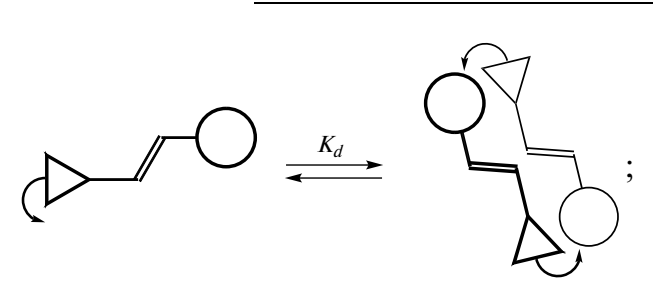


Here

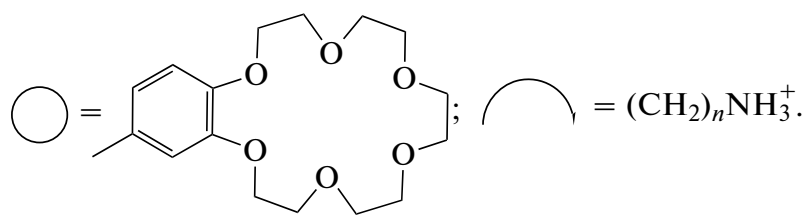

It was found that the chromophore fragments of two molecules in these complexes are located close to each other and are practically parallel to each other, while the mutual orientation of the central $\mathrm{C}=\mathrm{C}$ bonds is favorable for the $[2+2]$ photocycloaddition reaction [6]. It is not surprising that in this case it was possible to carry out stereospecific $[2+2]$ photocycloaddition reactions in solution with unusually high quantum yields.

The discovered property makes it possible to count on the use of these new photoswitchable supramolecular devices in the technology of optical recording and storage of information [6]:

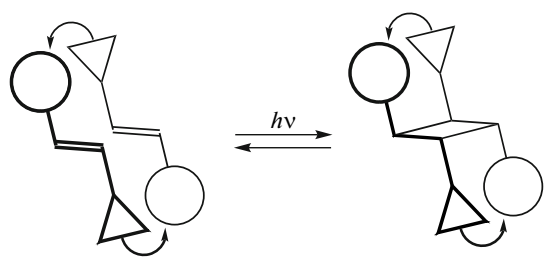

The quantum yield

of the photocycloaddition is 0.38

Self-assembly of photocontrolled supramolecular machines based on photoactive unsaturated compounds and cucurbiturils has become an important area of research [4, 5]. It turned out that cucurbiturils form rather stable inclusion complexes of the guest-host type with positively charged unsaturated compounds.

It was of interest to investigate the possibility of the photocontrol of mechanical movements in supramolecular machines. The simplest version of such a supramolecular machine was created based on the inclusion complex of naphthylpyridine (NP) with $\beta$-cyclodextrin. In this case, photoirradiation leads to reversible protonation and, as a consequence, to the mechanical displacement of the guest molecule in the macrocycle cavity:

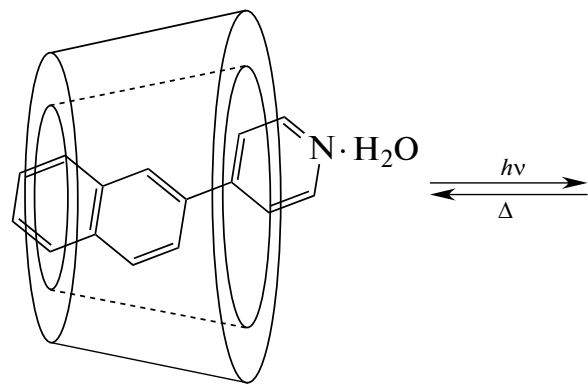

$\mathrm{H \Pi} \cdot \mathrm{H}_{2} \mathrm{O} @ \mathrm{HP}-\beta-\mathrm{CD}$

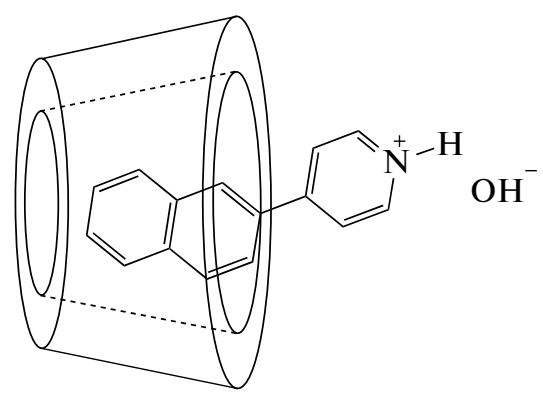

HП $\mathrm{H}^{+} \mathrm{OH}^{-} @ \mathrm{HP}-\beta-\mathrm{CD}$
The simplest fast photocontrolled supramolecular machine was obtained based on the inclusion complex of styryl dye and cucurbit[7]uril. It turned out that a significant increase in the lifetime of the dye's fluores- cence is related to the mechanical displacement of the electronically excited dye of the cation deep into the cavity of the host molecule in the first few picoseconds: 


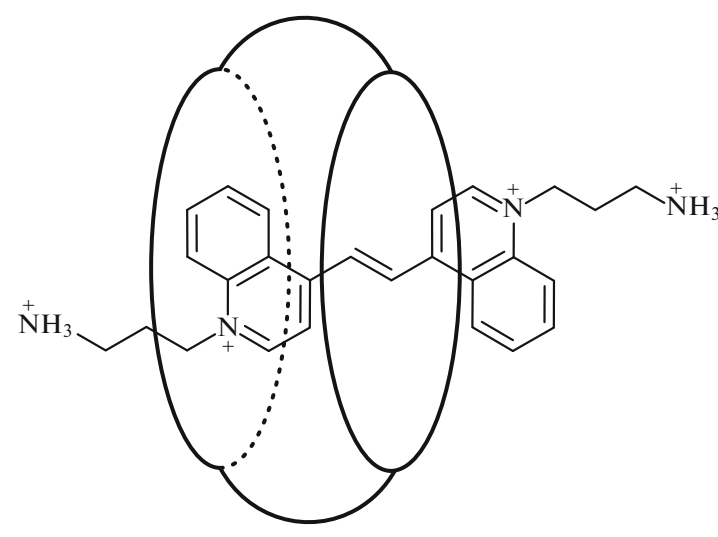

Pseudorotaxan trans-isomer inclusion complex

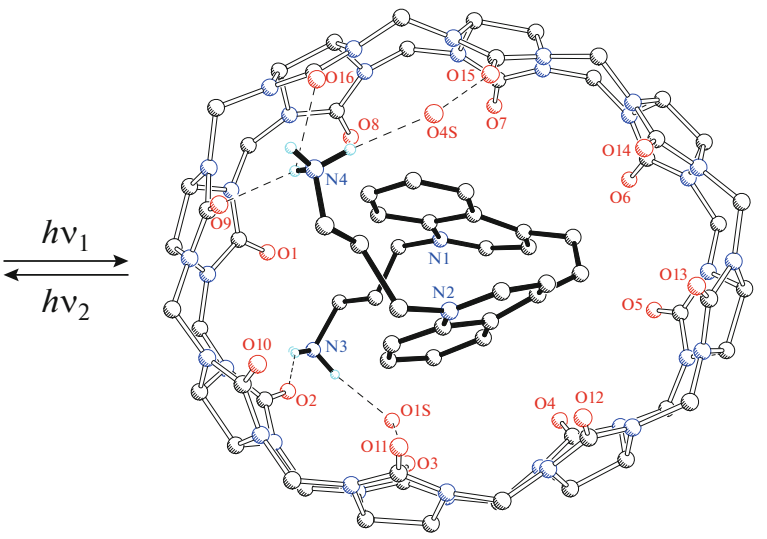

Cis-isomer inclusion complex

Fig. 3. Photocontrolled supramolecular machine based on pseudorotaxane complex of inclusion of a dichinolylethylene derivative with cucurbit[8]uril.
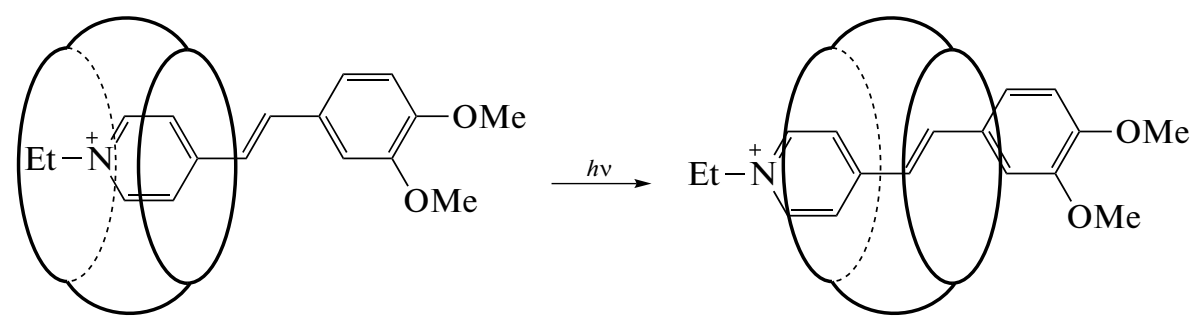

A more complex version of a photocontrolled supramolecular machine was created based on the pseudorotaxane complex of cucurbit[8]uril with a dichinolylethylene derivative. In this case, irradiation with light causes a reversible trans-cis-photoisomerization of the guest molecule of unsaturated compounds (Fig. 3) [7]. The spatial structure of this supramolecular machine after irradiation with light, determined by X-ray diffraction analysis (see the right side of Fig. 3), shows that the complex mechanical movement of the guest molecule in the cavity of the host macrocycle, reminiscent of coiling into a ball, can easily be realized.

It turned out that the cavity of cucurbit[8]uril can accommodate two styryl dye molecules. Figure 4 shows the structure of the corresponding supramolecular pseudorotaxane inclusion complex [8]. The mutual arrangement of unsaturated fragments of two dye molecules in this complex turned out to be favorable for the photocycloaddition reaction. The studies have shown that the resulting cyclobutane is less strongly bound to cucurbituril than the initial components; i.e., it becomes possible to control not only the mechanical movements of photoactive molecules within the macrocycle but also the strength with which the components are bound.
Due to these properties, it was possible to create a photocontrolled supramolecular assembler based on cucurbit[8]uril [8]. Assemblers are supramolecular machines capable of directing chemical reactions by positioning molecules (i.e., the guest molecules are the moving part of the mechanism in this case). In nature, the ribosome is an example of an assembler designed for the synthesis of cellular proteins from amino acids. In our case, the presence of only $5 \mathrm{~mol} \%$ of cucurbituril allows the complete stereospecific transformation of the initial styryl dyes into cyclobutane derivatives upon irradiation with light (the functioning of the photocontrolled supramolecular assembler is shown in Fig. 5).

Thus, using cucurbituril, it is possible to collect trimolecular pseudorotaxane complexes, carry out a photocycloaddition reaction in these complexes, and repeat this cycle of transformations the required number of times.

All the main types of photoprocesses can be realized in the created supramolecular systems $[1,3,5,9$, 10]. All this allows us to suggest the creation of a molecular meccano of photoactive supramolecular systems based on unsaturated and macrocyclic compounds, which has a unique set of the required characteristics [1]: (1) accessibility from the point of view of organic synthesis; (2) the tendency for spontaneous 


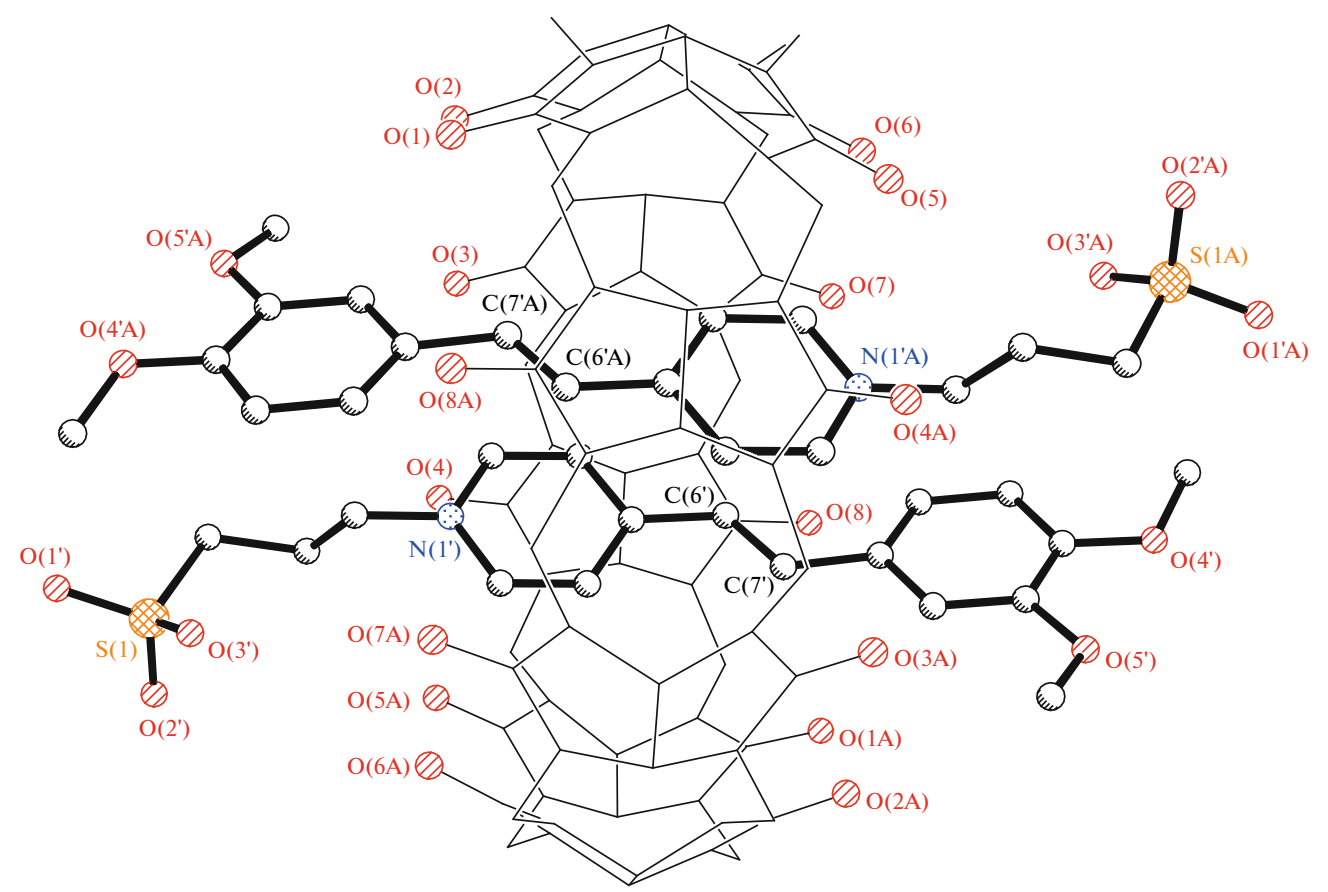

Fig. 4. Arrangement of two styryl dye molecules in the cucurbit[8]uril cavity in the pseudorotaxan inclusion complex.<smiles>CC[n+]1ccc(/C=C/c2cc(/C=C/c3ccc(OC)c(OC)c3)cc[n+]2CC)cc1</smiles>

Styryl dye (SD)

Cyclobutane (C)

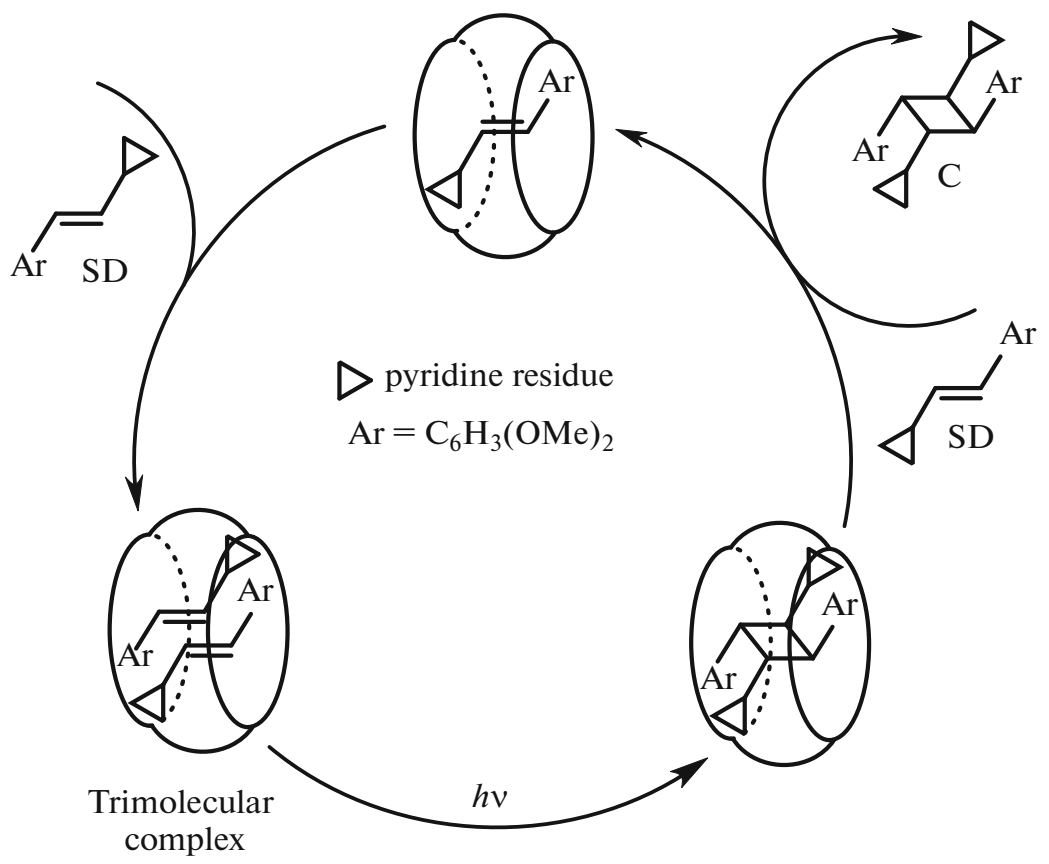

Fig. 5. Photocontrolled supramolecular assembler based on cucurbit[8] uril for the assembly of cyclobutane derivatives from styryl dyes. 
self-organization into various supramolecular architectures; (3) the property, depending on the structure, to undergo various types of photochemical transformations; and (4) the ability for efficient molecular photoswitching. These characteristics make it possible to collect in a solution, at the interface between the phases, and in the solid phase, photoswitchable supramolecular devices, photocontrolled supramolecular machines, and photoactive supramolecular systems with the given architecture and different properties.

Attention should be paid to the applied potential of the studies carried out, since they provide a new methodology for the construction of materials for supramolecular and nanophotonics, which was demonstrated primarily by the example of the creation of photoswitchable supramolecular devices, photocontrolled supramolecular machines, supramolecular switches, optical chemosensor materials, media for optical recording, and storage of information [3-5].

The results presented in this review were obtained mainly at the Center for Photochemistry of the Russian Academy of Sciences. At the same time, a number of Russian research organizations and universities in Sweden, the United States, Germany, Britain, and Italy took part in these studies.

In 2018, the authors of this study were awarded a state prize of the Russian Federation in Science and Technology for the development of photoactive supramolecular devices and machines.

\section{FUNDING}

The research was carried out with financial support from the Russian Science Foundation, the Russian Foundation for Basic Research, the Russian Academy of Sciences, the Ministry of Education and Science of the Russian Federation, the Moscow Government, the foundations of the Royal Society-INTAS, CRDF, and DFG-and the Soros Foundation.

The review was prepared with the financial support of the Ministry of Science and Higher Education as part of a state order of the Federal Research Center "Crystallography and Photonics" of the Russian Academy of Sciences.

\section{OPEN ACCESS}

This article is licensed under a Creative Commons Attribution 4.0 International License, which permits use, sharing, adaptation, distribution and reproduction in any medium or format, as long as you give appropriate credit to the original author(s) and the source, provide a link to the Creative Commons license, and indicate if changes were made. The images or other third party material in this article are included in the article's Creative Commons license, unless indicated otherwise in a credit line to the material. If material is not included in the article's Creative Commons license and your intended use is not permitted by statutory regulation or exceeds the permitted use, you will need to obtain permission directly from the copyright holder. To view a copy of this license, visit http://creativecommons.org/licenses/by/4.0/.

\section{REFERENCES}

1. S. P. Gromov, Russ. Chem. Bull. 57, 1325 (2008).

2. S. P. Gromov and M. V. Alfimov, Russ. Chem. Bull. 46, 611 (1997).

3. E. N. Ushakov, M. V. Alfimov, and S. P. Gromov, Russ. Chem. Rev. 77, 39 (2008).

4. S. P. Gromov, Obzor. Zh. Khim. 1 (1), 3 (2011).

5. E. N. Ushakov and S. P. Gromov, Russ. Chem. Rev. 84, 787 (2015).

6. S. P. Gromov, A. I. Vedernikov, N. A. Lobova, et al., Org. Chem. 79, 11416 (2014).

7. L. G. Kuz'mina, A. I. Vedernikov, N. A. Lobova, et al., New J. Chem. 30, 458 (2006).

8. S. P. Gromov, A. I. Vedernikov, L. G. Kuz'mina, et al., Eur. J. Org. Chem., No. 13, 2587 (2010).

9. A. K. Chibisov, S. V. Shvedov, and H. J. Goerner, J. Photochem. Photobiol., A 141, 39 (2001).

10. A. K. Chibisov and G. V. Zakharova, Photochem. Photobiol. Sci. 11, 893 (2012). 